\title{
INTERSECTION OF IDEALS WITH NON-COMMUTATIVE SUBALGEBRAS
}

\author{
VIKTOR LEVANDOVSKYY
}

\begin{abstract}
Computation of an intersection of a left ideal with a subalgebra, which is not fully investigated until now, is important for different areas of mathematics.

We present an algorithm for the computation of the preimage of a left ideal under a morphism of non-commutative $G R$-algebras, and show both its abilities and limitations.

The main computational tools are the elimination of variables by means of Gröbner bases together with the constructive treatment of opposite algebras and the utilization of a special bimodule structure.
\end{abstract}

KEYWORDS: Non-commutative algebra, Groebner bases, elimination, intersection with subalgebra, preimage of ideal, homomorphism of algebras, restriction.

\section{INTRODUCTION}

An intersection of a left or a two-sided ideal with the subalgebra of a given algebra is one of the fundamental constructions in algebra and its applications (e.g. in theoretical physics). The computation of the preimage of a twosided ideal reduces to the computation of the kernel of induced map both in the commutative (e.g. 8]) and the non-commutative cases ([2, 7, 20]).

On the contrary, the algorithmic treatment of the problem of computing the preimage of a left ideal, to the best of our knowledge, was only investigated in the non-commutative case for the situation of pure elimination ([2, 3, 7, 17, 20]). That is, one considered intersections only with subalgebras, generated by some subset of the set of variables.

In this article, we describe algorithms for computing the intersection of a two-sided and a left ideal with the finitely generated subalgebra of a noncommutative $G R$-algebra (see Def. 1) for various settings. We describe one of the possible ways to treat the opposite algebra of a $G R$-algebra constructively, and use this in further algorithms.

We start with subalgebras, generated by a subset of the set of variables of the algebra, closely investigate the notion of elimination of variables in $G^{-}$ algebras, and give explicit conditions for its computability. It is closely connected to Gröbner bases, so we review different definitions of them relatively to $G$-algebras. We are going to clarify the connection between Gröbner bases and filtrations on corresponding modules (Remark 3 ).

Then, we proceed with the general situation of an arbitrary finitely generated non-commutative subalgebra, and show the conditions to be satisfied

partially supported by the FWF (SFB grant F1301). 
for a map of algebras in order to be a morphism. After that, we present the method for computing the kernel of a morphism and hence, also the preimage of a two-sided ideal.

The algorithm for the preimage of a left ideal under a morphism of $G R$ algebras (Algorithm 1) is more complicated. We show that this algorithm works well for many examples, but it also has some natural limitations, which we discuss in detail. We comment the cases, which are important for applications, when a source or a target algebra is commutative.

$G R$-algebras, Gröbner bases and Gröbner basics for modules over $G R$ algebras are implemented as a subsystem PLURAL of the Computer Algebra System Singular (9]). Starting from the version 3-0-0 on, the Singular distribution includes PLURAL as an integral part; it is available for download at http://www.singular.uni-kl.de. All the examples in this article have been computed with this system.

By $\mathbb{K}$ we denote a field. In addition, we use the following notations:

$[a, b]=a \cdot b-b \cdot a$, a Lie bracket for polynomials $a, b$

${ }_{A}\langle S\rangle$, a left $A$-module, generated by a set $S$,

${ }_{A}\langle S\rangle_{B}$, a $(A, B)$-bimodule, generated by a set $S$,

$\mathbb{K}\langle S \mid R\rangle=\mathbb{K}\langle S\rangle /_{\mathbb{K}\langle S\rangle}\langle R\rangle_{\mathbb{K}\langle S\rangle}$, a presentation of a $\mathbb{K}$-algebra via the set of generators $S$ and the set of relations $R$,

$\mathrm{NF}(F \mid G)=\{\operatorname{NF}(f \mid G) \mid f \in F\}$, a normal form of a finite set $F$ with respect to a Gröbner basis $G$,

$x \gg y, x$ is bigger than any power of $y$.

\section{GR-ALGEBRAS}

Let $\mathbb{K}$ be a field, and $T=T_{n}=\mathbb{K}\left\langle x_{1}, \ldots, x_{n}\right\rangle$ a free associative $\mathbb{K}$-algebra, generated by $\left\{x_{1}, \ldots, x_{n}\right\}$ over $\mathbb{K}$.

Among the monomials $x_{i_{1}} x_{i_{2}} \ldots x_{i_{s}}, 1 \leq i_{1}, i_{2}, \ldots, i_{s} \leq n$, spanning $T$ as vector space over $\mathbb{K}$, we distinguish the standard monomials $x_{i_{1}}^{\alpha_{1}} x_{i_{2}}^{\alpha_{2}} \ldots x_{i_{m}}^{\alpha_{m}}$, where $1 \leq i_{1}<i_{2}<\ldots<i_{m} \leq n$ and $\alpha_{k} \in \mathbb{N}$. Via the correspondence $x^{\alpha}:=x_{1}^{\alpha_{1}} x_{2}^{\alpha_{2}} \ldots x_{n}^{\alpha_{n}} \mapsto\left(\alpha_{1}, \alpha_{2}, \ldots, \alpha_{n}\right)=: \alpha$ the set of standard monomials is in bijection with $\mathbb{N}^{n}$.

Recall, that any finitely generated associative $\mathbb{K}$-algebra is isomorphic to $T_{n} / I$, for some $n$ and some proper two-sided ideal $I \subset T_{n}$. If the set of standard monomials forms a $\mathbb{K}$-basis of an algebra $A=T / I$, we say that $A$ has a Poincaré-Birkhoff-Witt (shortly, PBW) basis in the variables $x_{1}, \ldots, x_{n}$.

As one can immediately see, the commutative polynomial ring $\mathbb{K}\left[x_{1}, \ldots, x_{n}\right]$ does have a PBW basis, while the free associative algebra $\mathbb{K}\left\langle x_{1}, \ldots, x_{n}\right\rangle$ does not. The existence of a PBW basis is an important property for a noncommutative algebra. However, we need more assumptions on the particular basis and the relations of an algebra in order to guarantee nice properties. In particular, the algebra $\mathbb{K}\langle x, y\rangle /\langle y x\rangle$ has a PBW basis, but it also has zero divisors. 
A total ordering $\prec$ on $\mathbb{N}^{n}$ is called a monomial ordering on the algebra $A$ with the PBW basis $\left\{x^{\alpha} \mid \alpha \in \mathbb{N}^{n}\right\}$, if $\forall \alpha, \beta, \gamma \in \mathbb{N}^{n}, \alpha \prec \beta \Rightarrow x^{\alpha} \prec x^{\beta} \Rightarrow$ $x^{\alpha+\gamma} \prec x^{\beta+\gamma}$. For $f \in T$, we denote by $\operatorname{lm}(f)$ the leading monomial of $f$ with respect to $\prec$.

Definition 1. Let $\mathbb{K}$ be a field, $T=\mathbb{K}\left\langle x_{1}, \ldots, x_{n}\right\rangle$ and $I$ be a two-sided ideal of $T$, generated by the elements

$$
x_{j} x_{i}-c_{i j} \cdot x_{i} x_{j}-d_{i j}, 1 \leq i<j \leq n,
$$

where $c_{i j} \in \mathbb{K} \backslash\{0\}$ and every $d_{i j} \in T$ is a polynomial, involving only standard $f^{1}$ monomials of $T$. A $\mathbb{K}$-algebra $A=T / I$ is called $\boldsymbol{a} G$-algebra, if the following conditions hold

\section{- Ordering condition:}

there exists a monomial well-ordering $\prec$ on $\mathbb{N}^{n}$ such that $\forall 1 \leq$ $i<j \leq n \operatorname{lm}\left(d_{i j}\right) \prec x_{i} x_{j}$.

\section{- Non-degeneracy condition:}

$\forall 1 \leq i<j<k \leq n$, to the sets $\left\{c_{i j}\right\}$ and $\left\{d_{i j}\right\}$ we associate a polynomial $N D C_{i j k}=c_{i k} c_{j k} \cdot d_{i j} x_{k}-x_{k} d_{i j}+c_{j k} \cdot x_{j} d_{i k}-c_{i j} \cdot d_{i k} x_{j}+$ $d_{j k} x_{i}-c_{i j} c_{i k} \cdot x_{i} d_{j k}$. A condition is satisfied, if each $N D C_{i j k}$ reduces to zero with respect to the generators of $I$.

$G$-algebras, and Gröbner bases for them, were introduced by Apel in [1]. They are also known from [19], and appear as algebras of solvable type in [10, 11, 17] and as PBW algebras in [3]. We have reported on the further progress on $G$-algebras, on the Gröbner bases theory for modules over them, and on implementation in [12, 14, 15.

It is important to mention, that any polynomial $N D C_{i j k}$ is equal to the so-called associator $\left(x_{k} \star x_{j}\right) \star x_{i}-x_{k} \star\left(x_{j} \star x_{i}\right)$ for the multiplication $\star$, defined as follows: $x_{j} \star x_{i}=x_{j} x_{i}$, if $j \leq i$ and $x_{j} \star x_{i}=c_{i j} x_{i} x_{j}+d_{i j}$, if $j>i$. As it has been pointed by a referee, the associator is one of the most important objects in the non-associative algebra. This motivates us to study the connection between $G$-algebras and Akivis algebras ([21]), which will be investigated in the future.

The PBW Theorem (from e.g. [14]) generalizes the classical PoincaréBirkhoff-Witt Theorem from the case of universal enveloping algebras of finite dimensional Lie algebras to the case of general $G$-algebras. Hence, a $G$-algebra in variables $x_{1}, \ldots, x_{n}$ has a canonical PBW basis $\left\{x_{1}^{\alpha_{1}} x_{2}^{\alpha_{2}} \ldots x_{n}^{\alpha_{n}} \mid \alpha_{k} \in \mathbb{N}\right\}$.

Theorem 1. Let $A$ be a $G$-algebra in $n$ variables. Then

1) $A$ is left and right Noetherian,

2) $A$ is an integral domain,

3) A is Auslander-regular and Cohen-Macaulay,

4) the Gel'fand-Kirillov dimension $\operatorname{GKdim}(A)=n$,

5) the global homological dimension $\operatorname{gl} \operatorname{dim}(A) \leq n$,

6) the Krull dimension $K r \cdot \operatorname{dim}(A) \leq n$.

\footnotetext{
$1_{\text {we assume this for simplicity of presentation }}$
} 
We refer to [18] for corresponding definitions, and to [6, 12] for definitions and proofs. There are examples (e.g. in [12]), where the inequalities 5) and 6) are strict.

We regard a $G$-algebra in $n$ variables as a generalization of a commutative polynomial ring in $n$ variables, taking into account, among others, the properties above. In particular, 1) and 2) imply that every $G$-algebra has a left and a right quotient ring.

For $G$-algebras $A$ and $B, A \otimes_{\mathbb{K}} B$ is a $G$-algebra. In the sequel, we use standard embeddings of $A$ and $B$ into $A \otimes_{\mathbb{K}} B$. We write $a$ resp. $b$ for the elements of $A \subset A \otimes_{\mathbb{K}} B$ resp. $B \subset A \otimes_{\mathbb{K}} B$, instead of $a \otimes 1$ resp. $1 \otimes b$, whenever no confusion is possible.

For a $G$-algebra $B$ and a proper nonzero two-sided ideal $I \subset B$, we call a factor algebra $B / I$ a $G R$-algebra.

For the constructive treatment of factor algebras, we need, in particular, two-sided Gröbner bases for two-sided ideals. One of the algorithmic approaches and its implementation were described in [15], whereas the authors of [5] propose a novel effective method for Gröbner bases of bimodules.

\section{Opposite Algebras}

Let $A$ be an associative algebra over $\mathbb{K}$. Recall, that the opposite algebra $A^{\text {opp }}$ is defined by taking the same vector-space as of $A$, and introducing a new "opposite" multiplication on it, that is $f * g:=g \cdot f$. Then, $A^{\text {opp }}$ is an associative $\mathbb{K}$-algebra, and $\left(A^{\text {opp }}\right)^{\text {opp }}=A$.

Lemma 1. Let $B=A / I$ be a GR-algebra. Then $B^{\text {opp }}$ is a GR-algebra, and $B^{\mathrm{opp}}=A^{\mathrm{opp}} / I^{\mathrm{opp}}$.

Opposite algebras are important, particularly due to the fact that for right-sided computations with a right module like a Gröbner basis, a syzygy module et cetera, it suffices to have a left-sided functionality implemented together with procedures for an effective treatment of opposite algebras and transfer of objects from an algebra into its opposite and back. The implementation in Singular:PluRAL is done along these lines.

For $p \in A$, we denote the opposed polynomial by $p^{*}$. As it was mentioned above, $\forall 1 \leq i<j<k \leq n N D C_{i j k}=\left(x_{k} \cdot x_{j}\right) \cdot x_{i}-x_{k} \cdot\left(x_{j} \cdot x_{i}\right)$.

In the opposite algebra, each corresponding non-degeneracy condition is of the form $\left(x_{i}^{*} * x_{j}^{*}\right) * x_{k}^{*}-x_{i}^{*} *\left(x_{j}^{*} * x_{k}^{*}\right)=0$. This inspires a particular construction of the opposite algebra, which we call the reversed $P B W$ basis method.

Let $X_{n+1-i}:=x_{i}^{*}$. Denote the induced monoid automorphism by $\sigma$ : $\mathbb{N}^{n} \rightarrow \mathbb{N}^{n}, \sigma(\alpha)=\sigma\left(\left(\alpha_{1}, \ldots, \alpha_{n}\right)\right):=\left(\alpha_{n}, \ldots, \alpha_{1}\right)$. Since a $\mathbb{K}$-basis of $A$ is the PBW basis

$\left\{x^{\alpha} \mid \alpha \in \mathbb{N}^{n}\right\}$, it is quite natural to define a monomial of $A^{\text {opp }}$ to be $\left(x^{\alpha}\right)^{*}:=X^{\sigma(\alpha)}=X_{n}^{\alpha_{n}} * \ldots * X_{1}^{\alpha_{1}}$. Hence, with this choice of monomial, the non-degeneracy condition is satisfied automatically.

Then, on $A^{\text {opp }}$ with the PBW basis $\left\{X^{\beta} \mid \beta \in \mathbb{N}^{n}\right\}$ there are relations, opposed to $A$, namely $\forall 1 \leq i<j \leq n, X_{i} X_{j}=C_{j i} X_{j} X_{i}+D_{j i}$. Define 
$C_{j i}:=c_{n+1-i, n+1-j}$ and $D_{j i}:=d_{n+1-i, n+1-j}^{*}$, then the pair $\left(X_{i}, X_{j}\right)$ together with the relation is clearly opposite to the pair $\left(x_{n+1-i}, x_{n+1-j}\right)$.

Let $M \in \mathbb{G} \mathbb{L}(n, \mathbb{R})$ be the matrix, representing an admissible well-ordering $\prec_{M}$ on $A$. Define a matrix $M^{*}$ by reverting the order of columns from $M=\left(M_{1}|\cdots| M_{n}\right)$ to $M^{*}=\left(M_{n}|\cdots| M_{1}\right)$. Note that $\prec_{M^{*}}$ is a wellordering if and only if $\prec_{M}$ is. Moreover, for any $\alpha \in \mathbb{N}^{n}, M \alpha=M^{*} \sigma(\alpha)$. Hence, we have the following: $x^{\alpha} \prec_{M} x^{\beta} \Leftrightarrow M \alpha \prec_{\text {lex }} M \beta \Leftrightarrow M^{*} \sigma(\alpha) \prec_{\text {lex }}$ $M^{*} \sigma(\beta) \Leftrightarrow X^{\sigma(\alpha)} \prec_{M^{*}} X^{\sigma(\beta)}$.

Then, from $\operatorname{lm}\left(d_{i j}\right) \prec_{M} x_{i} x_{j}$ it follows, that $\operatorname{lm}\left(D_{j i}\right) \prec_{M^{*}} X_{j} X_{i}$, and the ordering condition is satisfied. Hence, $A^{\text {opp }}$ is a $G$-algebra in $n$ variables by the Def. 1 .

The implementation in Singular:PluRal uses this method. We provide the function opposite ( $r$ ing $\mathrm{R}$ ), which constructs the opposite algebra $R^{\text {opp }}$ from a given $G$-algebra $R$. The following convention is used: we change the letters in corresponding names of variables (given by strings) into capitals and vice versa. For example, the variables $\{y 2, D x\}$ of $A$ will become the variables $\{Y 2, d X\}$ of $A^{\text {opp }}$.

Moreover, the function oppose (P), applied to an object $P$ (of the type polynomial/vector/ideal/module/matrix) of $R$, creates the opposite object $P^{\text {opp }}$ in the opposite algebra $R^{\text {opp }}$. In the Singular:PluRAL documentation and in [12], one finds more explanations and examples.

The following important conjecture is still open.

Conjecture 2. For a $G$-algebra $A$, there is an isomorphism of $\mathbb{K}$-algebras

$$
A \cong A^{\text {opp }} \text {. }
$$

\section{Gröbner Bases And Elimination}

Using the language, close to the one, used in the commutative case (e.g. [8]), the definition of a Gröbner basis carries over to submodules of free modules over $G$-algebras in its "commutative form", although the meaning of some properties may be different.

\subsection{Variants of Gröbner Bases.}

Let $A$ be a $G$-algebra in $n$ variables. We say that a monomial of a free module $A^{r}$ (involving component $i$ ) is an element of the form $x^{\alpha} e_{i}$, where $\alpha \in \mathbb{N}^{n}$ and $e_{i}$ is the canonical $i$-th basis vector.

We say, that $m_{1}=x^{\alpha} e_{j}$ divides $m_{2}=x^{\beta} e_{k}$, and denote it by $m_{1} \mid m_{2}$, if $j=k$ and $\alpha_{i} \leq \beta_{i} \forall i=1 \ldots n$. Note, that it is rather a pseudo-division on $A$, since if $m_{1} \mid m_{2}$, then there exist $c \in \mathbb{K} \backslash\{0\}$, a monomial $p \in A$ and $q \in A^{r}$ such that $\operatorname{lm}(q) \prec m_{1}$ and $m_{2}=c \cdot p \cdot m_{1}+q$, where $q$ is in general nonzero.

From the properties of $G$-algebras it follows, that any $f \in A^{r} \backslash\{0\}$ can be written uniquely as $f=c_{\alpha} x^{\alpha} e_{i}+g$, with $c_{\alpha} \in \mathbb{K}^{*}$, and $x^{\beta} e_{j} \prec x^{\alpha} e_{i}$ for any nonzero term $c_{\beta} x^{\beta} e_{j}$ of $g$. Then we define

$$
\begin{aligned}
& \operatorname{lm}(f)=x^{\alpha} e_{i}, \quad \text { the leading monomial of } f, \\
& \operatorname{lc}(f)=c_{\alpha}, \quad \text { the leading coefficient of } f, \\
& \operatorname{le}(f)=(i, \alpha), \text { the leading exponent of } f .
\end{aligned}
$$


Note, that $\forall \alpha, \beta \in \mathbb{N}^{n}, \operatorname{lm}\left(x^{\alpha} x^{\beta}\right)=\operatorname{lm}\left(x^{\alpha+\beta}\right)=\operatorname{lm}\left(x^{\beta} x^{\alpha}\right)$.

Definition 2. Let $\prec$ be a monomial ordering on the free module $A^{r}, I \subset A^{r}$ a left submodule, and $G \subset I$ a finite subset. $G$ is called a left Gröbner basis of $I$ if and only if for any $f \in I \backslash\{0\}$ there exists $g \in G$, satisfying $\operatorname{lm}(g) \mid \operatorname{lm}(f)$.

However, we need a more concrete description of Gröbner bases. We can use e.g. the span of leading monomials.

Let us denote $\mathbb{N}_{r}:=\{1, \ldots, r\}$.

Definition 3. Let $S$ be any subset of $A^{r}$.

- We define $\mathcal{L}(S) \subseteq \mathbb{N}_{r} \times \mathbb{N}^{n}$ to be a $\mathbb{N}^{n}$-monoideal, generated by the leading exponents of the elements of $S, \mathcal{L}(S)=\mathbb{N}^{n}\langle(i, \alpha)| \exists s \in$ $S, \operatorname{le}(s)=(i, \alpha)\rangle$. We call $\mathcal{L}(S)$ a monoideal of leading exponents.

- $L(S)$, the span of leading monomials of $S$, is defined to be the $\mathbb{K}$-vector space, spanned by the set $\left\{x^{\alpha} e_{i} \mid(i, \alpha) \in \mathcal{L}(S)\right\} \subseteq A^{r}$.

Remark 3. By Dixon's Lemma, $\mathcal{L}(S)$ is finitely generated, i.e. there exist $\left(i_{1}, \alpha_{1}\right), \ldots,\left(i_{m}, \alpha_{m}\right) \in \mathbb{N}_{r} \times \mathbb{N}^{n}$, such that $\mathcal{L}(S)=\mathbb{N}^{n}\left\langle\left(i_{1}, \alpha_{1}\right), \ldots,\left(i_{m}, \alpha_{m}\right)\right\rangle$ as monoideal.

In general, for $S \subset A^{r}, L(S)$ is just a $\mathbb{K}$-vector subspace of $A$. Using the filtration by the monomial ordering on $A^{r}$ (see e.g. [3, 14] for details), we see that indeed, $L(S)$ can be considered as a $\mathbb{K}$-subspace of the algebra $\operatorname{Gr}_{\prec}(A)$. The set $\Lambda=\left\{(i, \alpha) \mid \exists f \in S: \operatorname{lm}(f)=x^{\alpha} e_{i}\right\} \subset \mathbb{N}_{r} \times \mathbb{N}^{n}$ is equal to $\mathcal{L}(S)$. Hence,

$$
\operatorname{Gr}_{\prec}(S)=\bigoplus_{(i, \alpha) \in \Lambda} \mathbb{K} x^{\alpha} e_{i}=L(S) .
$$

It follows that $L(S)$ is a $\mathrm{Gr}_{\prec}(A)$-module.

Hence, if $A \cong \operatorname{Gr}_{\prec}(A)$ as $\mathbb{K}$-algebras, $L(S)$ is an $A$-module. It means that, for quasi-commutative algebras ( $G$-algebras with $d_{i j}=0 \forall 1 \leq i<$ $j \leq n$, e.g. commutative algebras), we can define $L(S)$ equivalently as $L^{\prime}(S)={ }_{A}\langle\{\operatorname{lm}(f) \mid f \in S\}\rangle$ and call it a leading submodule of $S$ (clearly, $L(S)=L^{\prime}(S)$ as $\mathbb{K}$-vector spaces). Then, a finite set $S$ is a Gröbner basis of ${ }_{A}\langle S\rangle$ if and only if $L^{\prime}(S)=L^{\prime}\left({ }_{A}\langle S\rangle\right)$.

The following folklore example shows, that the definition of Gröbner basis via leading submodules cannot be transferred directly to the case of general $G$-algebras.

Example 4. Consider the first Weyl algebra $A=\mathbb{K}\langle x, \partial \mid \partial x=x \partial+1\rangle$, the set $S=\{x \partial+1, x\}$, and the ideal $I={ }_{A}\langle S\rangle$. I is a proper left ideal equal to ${ }_{A}\langle x\rangle$, with $\{x\}$ a reduced Gröbner basis of $I$.

Hence, the $\mathbb{K}$-vector spaces $L^{\prime}(I)$ and ${ }_{A}\langle x\rangle$ are equal, but

$$
L^{\prime}(S)={ }_{A}\langle\{x \partial, x\}\rangle=A \cdot 1
$$

In view of the remark, we can give an alternative description of the Gröbner basis property. 
Definition 4. Let $\prec$ be a monomial ordering on the free module $A^{r}, I \subset A^{r}$ a left submodule, and $G \subset I$ a finite subset. $G$ is called a left Gröbner basis of $I$ if and only if the following equivalent conditions are satisfied:

- $L(G)=L(I)$ as $\mathbb{K}$-vector spaces,

- $\mathcal{L}(G)=\mathcal{L}(I)$ as $\mathbb{N}^{n}$-monoideals,

- $\mathbb{N}^{n}\langle\mathcal{L}(G)\rangle=\mathcal{L}(I)=\mathcal{L}\left({ }_{A}\langle G\rangle\right)$ as $\mathbb{N}^{n}$-monoideals.

\subsection{Elimination.}

The notion of elimination of variables can be transferred literally from commutative polynomial rings, where it has its historical origins, to associative algebras, having the following property:

every subset $S$ of the set of variables $X$ generates a subalgebra, not involving other variables than that of $S$.

In the sequel, we call such a subalgebra essential. It is easy to see, that many important algebras belong to the class of algebras, where all subalgebras, generated by any subset of the set of variables are essential. In addition to commutative polynomial rings, also free associative algebras, multiparameter quantum affine spaces (and more generally, quasi-commutative algebras) et cetera are in this class. On the other hand, there are still many algebras, which do not enjoy this property. In such algebras, we cannot speak of elimination of variables in general, but only of intersection with essential subalgebras. On the other side, "elimination" is terminologically so widespread and comfortable, that we may use it for all the cases, having in mind, however, an intersection with essential subalgebras.

Definition 5. (Elimination ordering) Let $A$ be a $G$-algebra, generated by $\left\{x_{1}, \ldots, x_{n}\right\}$, such that $\left\{x_{r+1}, \ldots, x_{n}\right\}$ generate an essential sub-G-algebra $B \subset A$. A monomial ordering $\prec$ on $A$ is an elimination ordering for $x_{1}, \ldots, x_{r}$, if for any $f \in A, \operatorname{lm}(f) \in B$ implies $f \in B$. If, moreover, $x_{1}, \ldots, x_{r}$ generate an essential sub-G-algebra $C$, we say in addition, that $\prec$ is an elimination ordering for $C$. We call such an ordering admissible elimination ordering $\prec_{A_{r}}$, if the condition $\forall i<j \operatorname{lm}\left(d_{i j}\right) \prec_{A_{r}} x_{i} x_{j}$ is satisfied. We use the notation $x_{m} \gg x_{k}$, if $x_{m} \in B$ and $x_{k} \notin B$.

A classical example of an elimination ordering for the definition above is the block ordering, composed of monomial orderings $\prec_{C}$ and $\prec_{B}$. The lexicographical ordering lex, widely used for elimination purposes in both commutative rings and free associative algebras, rarely satisfies the ordering condition of the Definition 1. However, lex is admissible for such important algebras like Weyl algebras, and a few other algebras.

A block ordering of the form $\left(\prec_{C}, \prec_{B}\right)$ is quite natural for use, but it is often not efficient in computations. Numerous experiments with concrete problems led us to the use of extra weight ordering (e.g. [8]) instead. Such an ordering seems to be generically the fastest elimination ordering 2 .

\footnotetext{
2 we must say, that the most complicated Gröbner bases computations that we dealt with, were elimination problems
} 
Suppose, we are in the situation of the Definition 5. Let $M \in \mathbb{G} \mathbb{L}(n, \mathbb{R})$ be the matrix, representing an admissible well-ordering $\prec_{M}$ on $A$. We put the row $\left(w_{1}, \ldots, w_{r}, 0, \ldots, 0\right)$ on the top of $M$, and throw away, say, the last row of $M$. The resulting matrix will be again in $\mathbb{G} \mathbb{L}(n, \mathbb{R})$. The positive weights $w_{i}$ are chosen in such a way, that the ordering condition of the Definition 1 is satisfied by $A$ with respect to the new ordering.

The computation of a tuple of strictly positive weights $\left(w_{1}, \ldots, w_{n}\right)$ for the given algebra $A$, such that $A$ is a $G$-algebra with respect to the wellordering with weight vector $\left(w_{1}, \ldots, w_{n}\right)$ can be achieved with the help of the method, described in e.g. [3]. This method is implemented as the procedure Gweights in the Singular:PluRAL library NCTOOLS.LIB ([16]). We are going to generalize this method to more general monomial orderings, including elimination orderings and implement it in PLURAL.

The following Lemma is the constructive formulation of the classical result of Gröbner bases theory, adopted for the $G$-algebras. In the proof we demonstrate the usefulness of the approach, introduced in the Def. 3 and Def. 4 (span of leading monomials).

Lemma 2. Let $A$ be a $G$-algebra, generated by $\left\{x_{1}, \ldots, x_{n}\right\}$ and $I \subset A$ be an ideal. Suppose, that the following conditions are satisfied:

- $\left\{x_{r+1}, \ldots, x_{n}\right\}$ generate an essential subalgebra $B$,

- there exists an admissible elimination ord. $\prec_{B}$ for $x_{1}, \ldots, x_{r}$ on $A$.

Then, if $S$ is a left Gröbner basis of I with respect to $\prec_{B}$, we have $S \cap B$ is a left Gröbner basis of $I \cap B$.

Proof. Take any $x^{\alpha} \in L(I)$, then there exists such $f \in I$, that $\operatorname{lm}(f)=x^{\alpha}$. Since $\prec_{B}$ is an elimination ordering for $x_{1}, \ldots, x_{r}$, from $\operatorname{lm}(f) \in B$ it follows that $f \in B$. Hence, $L(I) \cap B$ equals to $\oplus\left\{\mathbb{K} x^{\alpha} \mid \exists f \in I, \operatorname{lm}(f)=x^{\alpha}\right\} \cap B=$ $\oplus\left\{\mathbb{K} x^{\alpha} \mid \exists f \in I \cap B, \operatorname{lm}(f)=x^{\alpha}\right\}$, and the latter is just $L(I \cap B)$. Then $L(S) \cap B=L(I) \cap B=L(I \cap B)=L(S \cap B)$, hence, $S \cap B$ is a left Gröbner basis of $I \cap B$ by the Def. 4 .

Note, that both conditions we impose are automatically satisfied in a commutative polynomial ring and in a free non-commutative algebra. However, as the Lemma shows, one cannot transfer the method to arbitrary finitely presented associative algebras in a direct way. The generalization of the Elimination Lemma to a wider class of algebras is an interesting open problem.

The built-in command eliminate in Singular:PluRAL works along the lines of the Lemma 2. At first it checks whether $B$ is essential and, if it is the case, the check of the admissibility of an extra weight ordering with weights $(1, \ldots, 1)$ is performed. We plan to include the third component in this procedure, which will compute a vector of weights such that the corresponding extra weight elimination ordering is admissible, or report that no such weight vector exists.

In the next examples we are going to illustrate the crucial difference to the commutative elimination. Indeed, there are concrete situations, appearing in applications, where elimination requires extra computations. It may even happen that no elimination is possible. 
Example 5. (Elimination, requiring extra weights)

Consider the algebra $X=\mathbb{K}\langle a, b, x, d\rangle$ subject to relations $[b, a]=3 a$, $[d, a]=3 x^{2},[x, b]=-x,[d, b]=d,[d, x]=1$.

We fix a PBW basis $\left\{a^{p} b^{q} x^{r} d^{s}\right\}$. A small computation ensures, that the non-degeneracy condition on $X$ is satisfied. An admissible well-ordering $\prec$ on $X$ has to satisfy only one condition $x^{2} \prec a d$, which may be achieved by using a degree ordering. Since both $\{a, b\}$ and $\{x, d\}$ generate essential subalgebras, any block ordering, giving priority to $\{a, b\}$ and having degree orderings in every block, is an admissible elimination ordering for $\{a, b\}$.

Any elimination ordering for $\{x, d\}$ has to satisfy $x^{2} \prec d a$, while $x \gg a$ and $d \gg a$, which is impossible with standard block orderings with weights $(1, \ldots, 1)$. The ordering condition on weights is satisfied, as soon as $2 \operatorname{deg}_{\omega}(x) \leq \operatorname{deg}_{\omega}(a)+\operatorname{deg}_{\omega}(d)=\operatorname{deg}_{\omega}(d)$. For example, for the algebra $X$ with the PBW basis $\left\{x^{p} d^{q} a^{r} b^{s}\right\}$ the ordering with extra weights 1 resp. 3 for $x$ resp. $d$ is a possible solution.

Example 6. (No elimination is possible)

Let $A=\mathbb{K}\langle p, q \mid q p=p q+d(p, q)\rangle$ be a $G$-algebra for a fixed ordering $\prec$ (that is $\operatorname{lm}(d) \prec p q$ ). Then, if for some $m \geq 2, \operatorname{lm}(d)=q^{m}$, the intersection of any left ideal $I \subset A$ with the subalgebra $\mathbb{K}[p]$ cannot be computed, because of the following objection. The elimination ordering for such computation requires $q \gg p$, which implies $q^{m} \succ p q$, and hence $\operatorname{lm}(d) \succ p q$, which contradicts the ordering condition for $A$ as a $G$-algebra.

An explanation to the above fact may be the following. In the free associative algebra $T=\mathbb{K}\langle p, q\rangle$ consider the two-sided ideal $J$, generated by $f=q p-p q-q^{2}$. If, for some ordering $\prec$ on $T$, we have $\operatorname{lm}(f)=q p$ or $\operatorname{lm}(f)=p q$, then $\{f\}$ is a two-sided Gröbner basis of $J$. However, if $\operatorname{lm}(f)=q^{2}$, the two-sided Gröbner basis of $J$ is infinite, being equal to $\left\{p q^{n}+q p^{n}-n \cdot q p^{n-1} q \mid n \geq 1\right\}$. Hence, further computations with $J$ are ineffective.

\section{Preimage under a Morphism}

If we are going to find an intersection of an ideal with a subalgebra, being the homomorphic image of other $G R$-algebra, the best way to do this is to consider a map between two algebras, check whether this map is a morphism of $G R$-algebras and, if it is the case, compute the preimage of an ideal.

\subsection{Morphisms and Their Kernels.}

Let $A$ and $B$ be $G$-algebras. Suppose, there are proper two-sided ideals $T_{A} \subset A, T_{B} \subset B$, already given by their two-sided Gröbner bases, and there are $G R$-algebras $\mathcal{A}=A / T_{A}$ and $\mathcal{B}=B / T_{B}$.

We call a map $\Phi: \mathcal{A} \rightarrow \mathcal{B}$ a morphism of $G R$-algebras, if $\Phi$ is a homomorphism of $\mathbb{K}$-algebras, that is $\forall x, y \in \mathcal{A} \quad \Phi(x y)=\Phi(x) \cdot \Phi(y)$ and, moreover, $\Phi\left(T_{A}\right) \subseteq T_{B}$ holds.

A map $\Phi: \mathcal{A} \rightarrow \mathcal{B}$ is completely defined by its values on the generators $\left\{x_{i}\right\}$ of $\mathcal{A}$, that is $\Phi: x_{i} \mapsto p_{i}$, for $p_{i} \in \mathcal{B}$. 
The set of all morphisms $\Phi: \mathcal{A} \rightarrow \mathcal{B}$ between the $G R$-algebras $\mathcal{A}, \mathcal{B}$ is denoted by $\operatorname{Mor}(\mathcal{A}, \mathcal{B})$. Respectively, we denote by $\operatorname{Mor}(A, B)$ the set of morphisms between the $G$-algebras $\phi: A \rightarrow B$.

Lemma 3. Let $\phi: A \rightarrow B$ be the map between the $G$-algebras. Consider the set $X:=\{f-\phi(f) \mid f \in A\} \subseteq A \otimes_{\mathbb{K}} B$, naturally $\mathbb{K}$-spanned by $\left\{x^{\alpha}-\phi\left(x^{\alpha}\right) \mid\right.$ $\left.\alpha \in \mathbb{N}^{n}\right\}$, and another set $S=\left\{x_{i}-\phi\left(x_{i}\right) \mid 1 \leq i \leq n\right\} \subset A \otimes_{\mathbb{K}} B$. Then, there are the following inclusions of $\mathbb{K}$-vector-spaces:

$$
X \subset{ }_{A}\langle S\rangle_{\phi(A)} \subseteq{ }_{A}\langle S\rangle_{B} .
$$

Note, that $X$ itself carries no $A$-module structure in this context.

Theorem 7. Let $E^{o}:=A \otimes_{\mathbb{K}} B^{\text {opp }}, T_{E}^{o}:=T_{A}+T_{B}^{\text {opp }}$ a two-sided ideal and $\mathcal{E}^{o}:=\mathcal{A} \otimes_{\mathbb{K}} \mathcal{B}^{\text {opp }}=E^{o} /\left\langle T_{E}^{o}\right\rangle$ a GR-algebra. Define the set $S^{o}:=$ $\left\{x_{i}-\phi\left(x_{i}\right)^{\text {opp }} \mid 1 \leq i \leq n\right\} \subset E^{o}$. Then, the $(A, B)$-bimodule ${ }_{A}\langle S\rangle_{B}$ can be viewed as the left ideal $I_{\phi}^{o}:={ }_{A \otimes_{\mathbb{K}} B^{\mathrm{opp}}}\left\langle S^{o}\right\rangle$. Respectively, ${ }_{\mathcal{A}}\langle S\rangle_{\mathcal{B}}$ can be

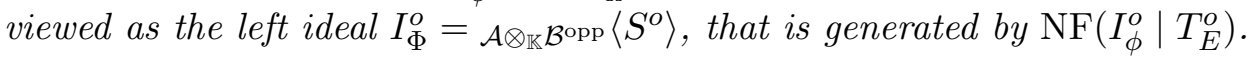
Then, the following holds:

(i) $\phi \in \operatorname{Mor}(A, B)$ if and only if $I_{\phi}^{o} \cap B^{o p p}=\langle 0\rangle$,

(ii) for any $\phi \in \operatorname{Mor}(A, B)$, $\operatorname{ker} \phi=I_{\phi}^{o} \cap A$,

(iii) $\Phi \in \operatorname{Mor}(\mathcal{A}, \mathcal{B})$ if and only if $\operatorname{NF}\left(I_{\phi}^{o} \cap B^{o p p} \mid I_{B}^{o p p}\right)=\langle 0\rangle$,

(iv) for any $\Phi \in \operatorname{Mor}(\mathcal{A}, \mathcal{B})$,

$$
\operatorname{ker} \Phi=I_{\Phi}^{o} \cap \mathcal{A}=\operatorname{NF}\left(T_{A}+\left(T_{B}^{o p p}+I_{\phi}^{o}\right) \cap A \mid T_{A}\right) .
$$

Example 8. Let $U\left(\mathfrak{s l}_{2}\right)$ be given in its standard presentation, namely, as $\mathbb{K}\langle e, f, h \mid[f, e]=-h,[h, e]=2 e,[h, f]=-2 f\rangle$. Moreover, let $W_{1}$ be the first Weyl algebra, already defined in Example 4. We consider the map

$$
\tau: U\left(\mathfrak{s l}_{2}\right) \rightarrow W_{1}, \tau(e)=x, \tau(f)=-x d^{2}, \tau(h)=2 x d .
$$

Using the reversed PBW basis method, we obtain $W_{1}^{\text {opp }}=\mathbb{K}\langle D, X|$ $X D=D X+1\rangle$. Let $E=U\left(\mathfrak{s l}_{2}\right) \otimes_{\mathbb{K}} W_{1}^{\text {opp }}$, and let $I_{\tau}^{o}$ be generated by $\left\{g_{1}=e-X, g_{2}=f+D^{2} X, g_{3}=h-2 D X\right\}$. Computing $I_{\tau}^{o} \cap W_{1}^{\text {opp }}$ gives zero: applying the Generalized Product Criterion ([15]), we see that $\operatorname{spoly}\left(g_{1}, g_{3}\right) \rightarrow-2 g_{1}, \operatorname{spoly}\left(g_{2}, g_{3}\right) \rightarrow 2 g_{2}$, and $\operatorname{spoly}\left(g_{1}, g_{2}\right) \rightarrow g_{3}$, where $\operatorname{spoly}(a, b)$ is a $s$-polynomial (see e.g. [3, 15, 17]) for the pair of polynomials $\{a, b\}$ and the reduction $\rightarrow$ is taken with respect to the set $\left\{g_{1}, g_{2}, g_{3}\right\}$.

Hence, $\left\{g_{1}, g_{2}, g_{3}\right\}$ is a left Gröbner basis with respect to an elimination ordering with $\{e, f, h\} \gg\{D, X\}$ and, indeed, $\tau \in \operatorname{Mor}\left(U\left(\mathfrak{s l}_{2}\right), W_{1}\right)$.

Let us compute the kernel of $\tau$. We set an elimination ordering with $\{D, X\} \gg\{e, f, h\}$ and compute the Gröbner basis of $I_{\tau}^{o}$ with respect to it. We obtain $\left\{4 e f+h^{2}-2 h, D h+2 f, 2 D e-h, X-e\right\} \subset E$ and see, that the polynomial $4 e f+h^{2}-2 h$ generates the kernel. Note, that this element is the generator of the center of $U\left(\mathfrak{s l}_{2}\right)$, hence, this element is the only generator of the two-sided Gröbner basis of $\operatorname{ker} \tau$. In particular, $\tau$ induces an injective morphism of $G R$-algebras

$$
0 \longrightarrow U\left(\mathfrak{s l}_{2}\right) /_{U\left(\mathfrak{s l}_{2}\right)}\left\langle 4 e f+h^{2}-2 h\right\rangle \stackrel{\tau}{\longrightarrow} W_{1} .
$$

Remark 9. With this technique we can compute preimages of two-sided ideals from $\mathcal{B}$ by computing the kernel of an induced morphism of factoralgebras. However, this does not allow us to compute the preimage of a left 
ideal from $\mathcal{B}$, since we transfer only the trivial $(A, A)$-bimodule structure on $A$ to $A \otimes_{\mathbb{K}} B^{\text {opp }}$. Namely, $\forall a, a^{\prime} \in A, b \in B^{\text {opp }}, a^{\prime} \circ(a \otimes b)=\left(a^{\prime} a \otimes 1\right) \cdot(1 \otimes b)$, and $(a \otimes b) \circ a^{\prime}=(1 \otimes b) \cdot\left(a a^{\prime} \otimes 1\right)$.

For a right ideal $L \subset B$, generated by $\left\{g_{1}, \ldots, g_{s}\right\}$, the left ideal $L^{o} \subset$ $A \otimes_{\mathbb{K}} B^{o p p}$ gets the left $A$-module structure and becomes the left $A \otimes_{\mathbb{K}} B^{o p p}$ module, generated by $\left\{1 \otimes g_{i}^{\mathrm{opp}}\right\}$. But then, $\left(L^{o}+I_{\phi}^{o}\right) \cap A$ is a left ideal instead of a right one, as the preimage must be.

\subsection{Algorithm for Preimage.}

For simplicity of the presentation, we formulate the following algorithm for $G$-algebras instead of $G R$-algebras.

Algorithm 1. NCPREIMAGE

Input 1: $A=\mathbb{K}\left\langle x_{1}, \ldots, x_{n} \mid\left\{x_{j} x_{i}=c_{i j} x_{i} x_{j}+d_{i j}\right\}\right\rangle$;

Input 2: $B=\mathbb{K}\left\langle y_{1}, \ldots, y_{m} \mid\left\{y_{j} y_{i}=a_{i j} y_{i} y_{j}+b_{i j}\right\}\right\rangle$;

Input 3: $J \subset B$ (left ideal);

Input 4: $\left\{f_{i}=\phi\left(x_{i}\right)\right\} \subset B$;

Output: $\phi^{-1}(J)$.

- $\forall 1 \leq i \leq m, \forall 1 \leq j \leq n$ define $q_{i j}:=\operatorname{lc}\left(y_{j} f_{i}\right) \cdot\left(\operatorname{lc}\left(f_{i} y_{j}\right)\right)^{-1}, q_{i j} \in \mathbb{K}^{*}$, and $r_{i j}:=y_{j} f_{i}-q_{i j} f_{i} y_{j} \in B$;

- search for an ordering, such that $\operatorname{lm}\left(r_{i j}\right) \prec x_{i} y_{j}$;

- if there exists no such ordering, report error;

- $E:=\mathbb{K}\left\langle y_{1}, \ldots, y_{m}, x_{1}, \ldots, x_{n}\right\rangle$ subject to relations $\left\{x_{j} x_{i}=c_{i j} x_{i} x_{j}+d_{i j}\right\}$,

$\left\{y_{j} y_{i}=a_{i j} y_{i} y_{j}+b_{i j}\right\},\left\{y_{j} x_{i}=q_{i j} x_{i} y_{j}+r_{i j}\right\}$;

- $I_{\phi}:=\left\{x_{i}-\phi\left(x_{i}\right) \mid 1 \leq i \leq n\right\} \subset E$;

- $P:=I_{\phi}+J \subset E$;

- $P=\operatorname{Eliminate}(P, B) ; \quad$ (that is $P:=P \cap A)$

- return $P$;

It is clear, that the algorithm terminates. The rest of the section is dedicated to the sketch of the proof of correctness.

Consider the additively closed set $G=\{g-\phi(g) \mid g \in A\} \subset A \otimes_{\mathbb{K}} B$. There is a natural left action of $A$ on $B$, induced by $\phi$, namely $a \circ_{L} b:=$ $\phi(a) b$. This action provides a well-defined left $A$-module structure on $B$, if $\forall a_{1}, a_{2} \in A, b \in B$, we have $a_{1} \circ_{L} a_{2} \circ_{L} b=\left(a_{1} \cdot a_{2}\right) \circ_{L} b$.

Indeed, $a_{1} \circ_{L} a_{2} \circ_{L} b-\left(a_{1} \cdot a_{2}\right) \circ_{L} b=\left(\phi\left(a_{1}\right) \phi\left(a_{2}\right)-\phi\left(a_{1} a_{2}\right)\right) b$, that is this action is well-defined if and only if $\phi$ is a morphism, which can be checked algorithmically with the Theorem 7. The natural right action is defined in an analogous way. Assume, that $\phi \in \operatorname{Mor}(A, B)$. Then $B$ is a $(A, A)-$ bimodule. Extending the action naturally to $A$ by $a_{1}{ }^{\circ} L a_{2}:=a_{1} \cdot a_{2}$, we turn $A \otimes_{\mathbb{K}} B$ into a $(A, A)$-bimodule.

Lemma 4. $G$ is a $(A, A)$-bimodule, generated by the set

$$
\left\{x_{i}-\phi\left(x_{i}\right) \mid 1 \leq i \leq n\right\} .
$$

The construction of the elimination algebra $E:=A \otimes_{\mathbb{K}}^{\phi} B$ in the algorithm is done for representing the described actions of $A$ on $A \otimes_{\mathbb{K}} B$. For this, we introduce the additional non-commutative relations between elements of $A$ and $B$. 
The action, written in terms of relations, gives $x_{i} y_{j}=f_{i} y_{j}, y_{j} x_{i}=y_{j} f_{i}$. Since $B$ is a $G$-algebra, $\operatorname{lm}\left(f_{i} y_{j}\right)=\operatorname{lm}\left(y_{j} f_{i}\right)$, and the corresponding relations become $\left\{y_{j} x_{i}=q_{i j} \cdot x_{i} y_{j}+r_{i j}\right\}$, where both $q_{i j}$ and $r_{i j}$ are already defined in the algorithm.

It remains to incorporate the relations $\left(x_{i}-f_{i}\right) y_{j}=0=y_{j}\left(x_{i}-f_{i}\right)$, $\forall 1 \leq i \leq n$ and $\forall 1 \leq j \leq m$. Since in $A \otimes_{\mathbb{K}}^{\phi} B, y_{j}\left(x_{i}-f_{i}\right)=\left(x_{i}-f_{i}\right) y_{j}$, it suffices to consider a two-sided ideal $R_{\phi} \subset A \otimes_{\mathbb{K}}^{\phi} B$, generated by $\left\{\left(x_{i}-f_{i}\right) y_{j} \mid\right.$ $\forall 1 \leq i \leq n, \forall 1 \leq j \leq m\}$. One possibility for treating the situation would be to pass to the factor algebra $A \otimes_{\mathbb{K}}^{\phi} B / R_{\phi}$. On the other hand, $R_{\phi} \subseteq G$ and in the computations below we need the sum $I_{\phi}+J$ and not $I_{\phi}:=G$ alone. The computation of a Gröbner basis of an ideal $J$ in the factor-algebra modulo $R_{\phi}$ is done by computing a Gröbner basis of the ideal $J+R_{\phi}$ and then, reducing the result with respect to $R_{\phi}$. Hence, the Gröbner basis of $I_{\phi}+J$ in the factor-algebra is the Gröbner basis of $I_{\phi}+J+R_{\phi}=I_{\phi}+J$, since $R_{\phi} \subseteq G$. Thus, $R_{\phi}$ can be skipped, since the action is already fully represented.

If we are given $G R$-algebras $\mathcal{A}, \mathcal{B}$, we construct $\mathcal{A} \otimes_{\mathbb{K}}^{\Phi} \mathcal{B}$ as a factor-algebra of $A \otimes_{\mathbb{K}}^{\phi} B$ by the two-sided ideal $T=T_{A}+T_{B}$.

Theorem 10. Let $\mathcal{A}, \mathcal{B}$ be $G R$-algebras, and $\Phi \in \operatorname{Mor}(\mathcal{A}, \mathcal{B})$.

Let $I_{\Phi}$ be the $(\mathcal{A}, \mathcal{A})$-bimodule $\mathcal{A}_{\mathcal{A}}\left\langle\left\{x_{i}-\Phi\left(x_{i}\right) \mid 1 \leq i \leq n\right\}\right\rangle_{\mathcal{A}} \subset \mathcal{A} \otimes_{\mathbb{K}} \mathcal{B}$, and $f_{i}:=\Phi\left(x_{i}\right)$. Suppose, there exists an elimination ordering for $B$ on $A \otimes_{\mathbb{K}} B$, such that $1 \leq i \leq n, 1 \leq j \leq m, \operatorname{lm}\left(\operatorname{lc}\left(f_{i} y_{j}\right) y_{j} f_{i}-\operatorname{lc}\left(y_{j} f_{i}\right) f_{i} y_{j}\right) \prec x_{i} y_{j}$. Then

1) $A \otimes_{\mathbb{K}}^{\phi} B$ is a $G$-algebra (resp. $\mathcal{A} \otimes_{\mathbb{K}}^{\Phi} \mathcal{B}$ is a $G R$-algebra).

2) Let $J \subset \mathcal{B}$ be a left ideal. Then

$$
\Phi^{-1}(J)=\left(I_{\Phi}+J\right) \cap \mathcal{A} .
$$

Proof. 1) Any elimination ordering for $A$ is admissible on $A \otimes_{\mathbb{K}}^{\phi} B$, since any such ordering has the property $x_{i} \succ y_{j}, r_{i j}$ depends only on $\left\{y_{k}\right\}$, and hence, $x_{i} y_{j} \succ \operatorname{lm}\left(r_{i j}\right)$. However, this will not always be the case for an elimination ordering for $B$ with its property $y_{j} \succ x_{i}$, thus, the condition of the theorem is essential.

The non-degeneracy condition is satisfied; we omit the corresponding very technical computation.

2) By Lemma 4, $\forall g \in A, g-\Phi(g) \in I_{\Phi}$. Since $\Phi$ is a morphism, and an elimination ordering for $A$ is admissible on $\mathcal{A} \otimes_{\mathbb{K}}^{\Phi} \mathcal{B}$, we have $I_{\Phi} \cap B^{\text {opp }} \subseteq T_{B}^{\text {opp }}$, and hence, $I_{\Phi} \cap J \subseteq I_{\Phi} \cap \mathcal{B}^{\text {opp }}=0$. Then $\left(I_{\Phi}+J\right) \cap \mathcal{B}=J$.

Since there exists an admissible elimination ordering for $B$, the intersection with $A$ is computable for an ideal in $A \otimes_{\mathbb{K}}^{\phi} B$. For any $q \in\left(I_{\Phi}+J\right) \cap \mathcal{A}$ we see, that $\Phi(q)=(\Phi(q)-q)+q \in\left(I_{\Phi}+J\right) \cap \mathcal{B}=J$, and the inclusion $\Phi^{-1}(J) \supset\left(I_{\Phi}+J\right) \cap \mathcal{A}$ holds.

Conversely, let $p \in \Phi^{-1}(J) \subset \mathcal{A}$. Then $p=p-\Phi(p)+\Phi(p) \in$ $\in\left(I_{\Phi}+J\right) \cap \mathcal{A}$.

The Algorithm 1 that we have presented works well for many interesting examples.

Example 11. Let $W_{1}$ be the first Weyl algebra $\mathbb{K}\langle x, d \mid[d, x]=1\rangle$. For $t \in \mathbb{Z}$ let $S_{t}=\mathbb{K}\langle a, b \mid[b, a]=t \cdot a\rangle$ be the universal enveloping algebra of a two-dimensional solvable Lie algebra. 
For a fixed $t \geq 2$, we consider the map $\psi_{t}: S_{t} \longrightarrow W_{1}$, $\psi_{t}(a)=x^{t}, \psi_{t}(b)=x d+t$. For $p \in \mathbb{N}$, let $I_{p}=W_{1}\left\langle x^{p}, x d+p\right\rangle$ and $J_{p}=$ $W_{1}\left\langle d^{p}, x d-p+1\right\rangle$ be left ideals. We are interested in preimages of left ideals $I_{p}, J_{p}$ under the map $\psi_{t}$.

Using the Theorem 7 , we conclude that $\forall t \in \mathbb{N}, \psi_{t}$ is a morphism. Moreover, based on the computations for various concrete $t$, we conjecture that $\forall t \in \mathbb{N}$, $\operatorname{ker} \psi_{t}=\langle 0\rangle$.

Now, we apply the Algorithm 1. In the algebra $E=S_{t} \otimes_{\mathbb{K}}^{\psi_{t}} W_{1}$, there will be the following new relations: $\left\{[x, b]=[x, x d+t]=-x,[d, a]=\left[d, x^{t}\right]=\right.$ $\left.t x^{t-1},[d, b]=[d, x d+t]=d\right\}$. Hence, the condition of the Theorem 10 is satisfied, as soon as $x^{t-1} \prec a d$ and $\{x, d\} \succ a$ hold at the same time.

Using the extra weight vector $(0,0,1, t)$, based on any well-ordering on variables $(a, b, x, d)$, we come to admissible elimination ordering on $S_{t} \otimes_{\mathbb{K}}^{\psi_{t}} W_{1}$. On the $W_{1} \otimes_{\mathbb{K}}^{\psi_{t}} S_{t}$, the extra weight vector $(1, t, 0,0)$ is applied to the variables $(x, d, a, b)$.

Let us compute some preimages for $t=7$. Computing with Plural, we obtain the following results:

$$
\begin{aligned}
& \psi_{7}^{-1}\left(I_{3}\right)=\langle a, b-4\rangle, \\
& \psi_{7}^{-1}\left(I_{8}\right)=\left\langle a^{2}, b+1\right\rangle, \\
& \psi_{7}^{-1}\left(I_{33}\right)=\left\langle a^{5}, b+26\right\rangle .
\end{aligned}
$$

From the experimental data, we conjecture that

$$
\forall t \in \mathbb{N}, \psi_{t}^{-1}\left(I_{p}\right)=\left\langle a^{\left[\frac{p}{t}\right]+1}, b+p-t\right\rangle .
$$

If it holds, we conclude that for any $p^{\prime} \in \mathbb{N}$ we have

$$
\psi_{t}\left(\psi_{t}^{-1}\left(I_{p}\right)\right)=\left\langle x^{p+p^{\prime}}, x d+p\right\rangle=\left\langle x^{p}, x d+p\right\rangle=I_{p} .
$$

The preimages of $J_{p}$ look more simple:

$\psi_{7}^{-1}\left(J_{3}\right)=\langle b-9\rangle$,

$\psi_{7}^{-1}\left(J_{8}\right)=\langle b-14\rangle$,

$\psi_{7}^{-1}\left(J_{33}\right)=\langle b-39\rangle$.

We may conjecture, that $\forall t \in \mathbb{N}, \psi_{t}^{-1}\left(J_{p}\right)=\langle b+1-(p+t)\rangle$.

If it holds, we conclude that $\psi_{t}\left(\psi_{t}^{-1}\left(J_{p}\right)\right)=\langle x d+1-p\rangle$, which is strictly contained in $J_{p}$.

In addition, let us compute the preimage of the ideal $K=I_{3} \cap J_{3}$. We get the following results: $K=\left\langle x^{4} d-2 x^{3}, x^{2} d^{2}+2 x d-6, x d^{4}+6 d^{3}\right\rangle$, $\psi_{7}^{-1}(K)=\left\langle a b-9 a, b^{2}-13 b+36\right\rangle=\langle a(b-9),(b-4)(b-9)\rangle$. As we can check e. g. with Plural, $\psi_{7}^{-1}(K)=\psi_{7}^{-1}\left(I_{3}\right) \cap \psi_{7}^{-1}\left(J_{3}\right)$.

In the following example we show, that the limitations of the method might also appear in applications in a natural way.

Example 12. Let us continue with the example 8. For the map $\tau: U\left(\mathfrak{s l}_{2}\right) \rightarrow W_{1}, \tau(e)=x, \tau(f)=-x d^{2}, \tau(h)=2 x d$, we build the algebra $E=U\left(\mathfrak{s l}_{2}\right) \otimes_{\mathbb{K}}^{\tau} W_{1}$, introducing new relations $\{[d, e]=1,[x, f]=2 x d,[d, f]=$ $\left.-d^{2},[x, h]=-2 x,[d, h]=2 d\right\}$. As we see, only two relations impose real restrictions: $f x \succ x d$ and $f d \succ d^{2}$, both being true if and only if $f \succ d$. But this is incompatible with the elimination ordering condition for $W_{1}$. Hence, the condition of the theorem is not fulfilled, and there is no way to compute preimages of left ideals under $\tau$ using this approach. However, 
we can use maps from different subalgebras of $U\left(\mathfrak{s l}_{2}\right)$ to $W_{1}$ and succeed with the Algorithm 1. In particular, we can use subalgebras $A_{e}$ and $A_{f}$ of $U\left(\mathfrak{s l}_{2}\right)$, generated by $\{e, h\}$ and $\{f, h\}$ respectively. Note, that $A_{e} \cong S_{2}$ and $A_{f} \cong S_{-2}$. Moreover, different morphisms $S_{t} \rightarrow A_{1}$ were already studied in the Example 11.

\subsection{Special Cases.}

If the source algebra $\mathcal{A}$ is commutative, then a map $\Phi: \mathcal{A} \rightarrow \mathcal{B}$ is a morphism if and only if the values of the generators of $A$ commute pairwise in $\mathcal{B}$. In this situation, there is an easier algorithm, than the Algorithm 1 , which is considered in detail in [13]. It is important to mention, that is this case many interesting questions arise. In particular, with the preimage algorithm we can determine the algebraic dependence of pairwise commuting elements, decide whether an element of $\mathcal{B}$ belongs to a commutative subalgebra of $\mathcal{B}$, and compute the central character decomposition of modules over $G R$ algebras. All of these applications are described in detail and illustrated by numerous examples in [13. Moreover, many algorithms of that article are implemented in Singular:PluRaL.

Some of the elimination problems in this setting lead to tremendous computations and may be used as benchmarks for high performance computer algebra systems. For example, consider the universal enveloping algebra $A$ of a finite dimensional simple Lie algebra over a field $\mathbb{K}$. Over any field, the center $Z(A)$, which is a subalgebra, is generated by pairwise commuting polynomials. However, in the case char $\mathbb{K}>0$, it is known from the dimension calculation, that these elements are algebraically dependent. There are several open questions on the dependency polynomials which we investigate by using computer algebraic methods.

We were able to compute the dependency polynomials explicitly for many prime $p$ over the algebras $U\left(\mathfrak{s l}_{2}\right)$ (see [12]) and $U\left(\mathfrak{s o}_{3}\right)$. Up to now, the case of $U\left(\mathfrak{s l}_{3}\right)$ remains unsolved and constitutes an important challenge for SiNGULAR:PLURAL.

If the target algebra $\mathcal{B}$ is commutative, and the source algebra $\mathcal{A}$ is noncommutative, a typical map $\Phi: \mathcal{A} \rightarrow \mathcal{B}$ is often not a morphism. However, in this situation the algorithm for computing the one-dimensional representations of $\mathcal{B}$ in $\mathbb{G} \mathbb{L}(1, \mathbb{K})=\mathbb{K}$ arises, see [12].

\section{Conclusions}

In the case of elimination, there may appear two difficulties. The first one lies in the fact, that in many algebras not every subset of the set of variables generate an essential subalgebra, hence it is not possible to "eliminate" certain group of variables. The second one arises in associative algebras, which have some condition on the monomial ordering used, like $G$-algebras in the Definition 1. More generally, this phenomenon may happen with a finitely presented associative algebra, which generators constitute a finite Gröbner basis with respect to some specific orderings and lead to infinite Gröbner 
basis for other orderings. In this case one has to look for an elimination ordering, satisfying the conditions (like finitness of Gröbner basis etc.) and, as it was demonstrated in the Example 6, it can happen that no such ordering exists. Summarizing, we see that it is not always possible to intersect ideals with certain subalgebras, even with very natural ones.

The Algorithm 1 provides a very general tool for computing preimages of left ideals of $G R$-algebras, and it is as complex as the Buchberger's algorithm with respect to an elimination ordering.

It is important to investigate, whether the ideas behind the Algorithm 1 can be constructively generalized to the different partial Ore localizations of $G$-algebras, like rational Weyl algebras

$\mathbb{K}\left(x_{1}, \ldots, x_{n}\right)\left\langle\partial_{1}, \ldots, \partial_{n} \mid\left[\partial_{i}, x_{i}\right]=1,\left[\partial_{j}, x_{k}\right]=0\right\rangle$

or local polynomial Weyl algebras

$\mathbb{K}\left[x_{1}, \ldots, x_{n}\right]_{\left\langle x_{1}, \ldots, x_{n}\right\rangle}\left\langle\partial_{1}, \ldots, \partial_{n} \mid\left[\partial_{i}, x_{i}\right]=1,\left[\partial_{j}, x_{k}\right]=0\right\rangle$.

More generally, there is a need for such results in classes of Ore algebras (4]) and even in very general PBW rings $([3,11])$. It is interesting to know, up to which extent these ideas may be generalized to the case of a morphism between two finitely presented associative algebras.

\section{ACKnOWledgments}

I would like to thank Gert-Martin Greuel, Christoph Lossen and Oleksandr Khomenko for enlightening discussions on the subject and for valuable remarks. The suggestions of anonymous referees helped to make the exposition more clear and complete. I wish to thank my wife Tetyana for helping to prepare the final version of this article.

The author is grateful to the Austrian FWF (SFB grant F1301) for the partial financial support.

\section{REFERENCES}

[1] Apel, J. Gröbnerbasen in nichtkommutativen Algebren und ihre Anwendung. Dissertation, Universität Leipzig, 1988.

[2] Borges, M. A. and Borges, M. Gröbner bases property on elimination ideal in the noncommutative case. In B. Buchberger and F. Winkler, editors, Gröbner bases and applications, pages 323-337. Cambridge University Press, 1998.

[3] Bueso, J., Gómez-Torrecillas, J. and Verschoren, A. Algorithmic methods in noncommutative algebra. Applications to quantum groups. Kluwer Academic Publishers, 2003.

[4] Chyzak, F. and Salvy, B. Non-commutative Elimination in Ore Algebras Proves Multivariate Identities. Journal of Symbolic Computation, 26(2):187-227, 1998.

[5] García Román, M. and García Román, S. Gröbner bases and syzygies on bimodules over PBW algebras. Journal of Symbolic Computation, 40(3):1039-1052, 2005.

[6] Gómez-Torrecillas, J. and Lobillo, F.J. Auslander-regular and Cohen-Macaulay quantum groups. J. Algebr. Represent. Theory, 7(1):35-42, 2004.

[7] Green, E. Multiplicative Bases, Gröbner Bases, and Right Gröbner Bases. Journal of Symbolic Computation, 29(4/5), 2000.

[8] Greuel, G.-M. and Pfister, G. with contributions by Bachmann, O., Lossen, C. and Schönemann, H. A SINGULAR Introduction to Commutative Algebra. Springer, 2002. 
[9] Greuel, G.-M., Pfister G., and Schönemann H. Singular 3.0. A Computer Algebra System for Polynomial Computations. Centre for Computer Algebra, University of Kaiserslautern, 2005. Available from http://www.singular.uni-kl.de.

[10] Kandri-Rody, A. and Weispfenning, V. Non-commutative Gröbner bases in algebras of solvable type. Journal of Symbolic Computation, 9(1):1-26, 1990.

[11] Kredel, H. Solvable polynomial rings. Shaker, 1993.

[12] Levandovskyy, V. Non-commutative computer algebra for polynomial algebras: Gröbner bases, applications and implementation. Doctoral Thesis, Universität Kaiserslautern, 2005. Available from http://kluedo.uni-kl.de/volltexte/2005/1883/.

[13] Levandovskyy, V. On preimages of ideals in certain non-commutative algebras. In Pfister G., Cojocaru S. and Ufnarovski, V., editor, Computational Commutative and Non-Commutative Algebraic Geometry. IOS Press, 2005.

[14] Levandovskyy, V. PBW Bases, Non-Degeneracy Conditions and Applications. In Buchweitz, R.-O. and Lenzing, H., editor, Representation of algebras and related topics. Proceedings of the ICRA X conference, volume 45, pages 229-246. AMS. Fields Institute Communications, 2005.

[15] Levandovskyy, V. and Schönemann, H. Plural - a computer algebra system for noncommutative polynomial algebras. In Proc. of the International Symposium on Symbolic and Algebraic Computation (ISSAC'03). ACM Press, 2003.

[16] Levandovskyy, V., Lobillo, F.J. and Rabelo, C. A Singular 3.0 library, providing general tools for noncommutative algebras nctools.lib. 2004.

[17] Li, H. Noncommutative Gröbner bases and filtered-graded transfer. Springer, 2002.

[18] McConnell, J.C. and Robson, J.C. Noncommutative Noetherian rings. With the cooperation of L. W. Small. Graduate Studies in Mathematics. 30. Providence, RI: American Mathematical Society (AMS), 2001.

[19] Mora, T. Groebner bases in non-commutative algebras. In Proc. of the International Symposium on Symbolic and Algebraic Computation (ISSAC'88), pages 150-161. LNCS 358, 1989.

[20] Nordbeck, P. On some basic applications of Gröbner bases in non-commutative polynomial rings. In B. Buchberger and F. Winkler, editors, Gröbner bases and applications, pages 463-472. Cambridge University Press, 1998.

[21] I. Shestakov and U. Umirbaev. Free Akivis algebras, primitive elements, and hyperalgebras. J. Algebra, 250(2):533-548, 2002.

Research Institute for Symbolic Computation (RISC), Johannes Kepler University, Altenbergerstrasse 69, A-4040 Linz, Austria

E-mail address: levandov@risc.uni-linz.ac.at 